Nota de investigación

\title{
Azúcares totales y proteína en frijol nativo de la región Triqui Alta, Oaxaca
}

\author{
Carmen Jacinto-Hernández ${ }^{1 \S}$ \\ Marisol Coria-Peña ${ }^{2}$ \\ Georgina Contreras-Santos ${ }^{2}$ \\ Liliana Martínez-López ${ }^{3}$ \\ Emma Zapata-Martelo ${ }^{3}$ \\ María del Rosario Ayala-Carrillo ${ }^{3}$ \\ ${ }^{1}$ Campo Experimental Valle de México-INIFAP. Carretera los Reyes-Texcoco km13.5, Coatlinchán, \\ Texcoco, Estado de México. AP. 10. CP. 56250. ${ }^{2}$ Tecnológico de Estudios Superiores de Chimalhuacán \\ (TESCHI). ${ }^{3}$ Colegio de Postgraduados-Campus Montecillo. Carretera México-Texcoco km 36.5, \\ Montecillo, Texcoco, Estado de México. CP. 56230. \\ ${ }^{\S}$ Autora para correspondencia: jacinto.carmen@inifap.gob.mx.
}

\section{Resumen}

La siembra de compuestos de frijol, que combinan tamaños y colores, es una tradición cultural en las zonas rurales de México, utilizados principalmente para autoconsumo. Las mezclas de frijol tienen un atractivo visual; sin embargo, poco se sabe de las características de sabor y valor nutrimental que representa esta forma de consumir el frijol. El objetivo fue conocer el contenido de azúcares totales y de proteína en compuestos de frijol nativo provenientes de la región Triqui Alta de Oaxaca. En el 2017 se realizó el estudio de tres muestras compuestas de semilla de frijol. El color de la testa se determinó visualmente y se midió el peso de 100 granos. El contenido de azúcares totales se determinó con el método de antrona, y el contenido de proteína con el equipo semiautomatizado Kjeltec. Los análisis se realizaron por duplicado. En cada muestra se identificaron al menos 4 diferentes colores y tamaños de grano identificados como frijol común (Phaseolus vulgaris L.) y ayocote (Phaseolus coccineus L.) El contenido de azúcares en los granos medianos fue de 6.17 a $6.48 \%$, mientras que, en los granos grandes (ayocote), fue desde 9.45 a 11.6\%. El contenido de proteína varió entre submuestras desde $21.7 \%$ en $P$. coccineus negro, hasta $24.8 \%$ en $P$. vulgaris amarillos. El color de grano no se asoció con los contenidos de proteína ni de azúcares totales; sin embargo, el tamaño de grano sí. A mayor tamaño de grano, mayor porcentaje de azúcares $\left(\mathrm{r}=0.88^{* *}\right)$ y menor contenido de proteína $\left(\mathrm{r}=-0.61^{*}\right)$.

Palabras clave: $P$. vulgaris, $P$. coccineus, color de la testa, compuestos de frijol.

Recibido: septiembre de 2019

Aceptado: octubre de 2019 
Dentro de los alimentos del grupo de leguminosas comestibles, el frijol es de los más importantes en México, por el bajo costo y ser complemento nutrimental indispensable en la dieta alimenticia; ya que la semilla aporta casi la totalidad de las proteínas vegetales, carbohidratos, fibra dietética, vitaminas y minerales que consume la mayoría de la población en el país (Gispert et al., 2004).

Por su valor nutrimental, el frijol es un alimento cuyo consumo debiera estimularse en la región Triqui, de acuerdo con el estudio realizado por Ramos y Mendoza (2007), los triquis, habitantes de la región Mixteca alta y baja, en el estado de Oaxaca han cambiado su dieta a un mayor consumo de alimentos industrializados de alta densidad calórica y bajo valor nutrimental, por lo que en el estado nutricional coexisten la desnutrición y la obesidad. Por otra parte, las mujeres presentan mayor probabilidad de tener baja estatura, lo que supone un riesgo para la gestación.

La desnutrición primaria (causa exclusivamente alimentaria), es muchos más frecuente en los niños y en las mujeres embarazadas o lactantes, se debe a una alimentación crónicamente insuficiente o mal estructurada, en la que la insuficiencia de energía se combina con deficiencia en el consumo de proteínas, hierro y algunas vitaminas y minerales (Bourges, 2001).

El frijol constituye una de las principales fuentes de proteína en la dieta de grandes segmentos de la población, principalmente en las zonas rurales y urbanas marginales. El contenido de proteína, en 65 genotipos nativos de frijol $P$. vulgaris del estado de Hidalgo varió desde 16.0\% (tipo ojo de cabra) hasta $26.9 \%$ (tipo negro). La proteína, al igual que otros factores de calidad es afectada por el ambiente (Muñoz-Velázquez et al., 2009).

En la región triqui, al igual que en otras zonas rurales de México se cultivan diferentes tipos de frijol nativo. En México existen diferentes especies de frijol que en ocasiones se siembran como compuestos que combinan diferentes tamaños y colores, lo cual, además de una tradición cultural, es una alternativa ante los factores ambientales adversos y asegura el alimento para las familias de los campesinos cuya producción es mayormente para autoconsumo y en ocasiones una parte se destina a la venta en mercados locales (Martínez-López, 2018).

La mezcla de tipos y colores de semillas de frijol resulta atractiva a la vista. Las antocianinas son pigmentos responsables de la gama de colores que abarcan desde el rojo hasta el azul de muchas frutas, vegetales y cereales. El interés en estos pigmentos se ha intensificado gracias a sus posibles efectos terapéuticos y benéficos, dentro de los cuales se encuentran la reducción de la enfermedad coronaria, los efectos anticancerígenos, antitumorales, antiinflamatorios y antidiabéticos; además del mejoramiento de la agudeza visual y del comportamiento cognitivo (Garzón, 2008). Se ha

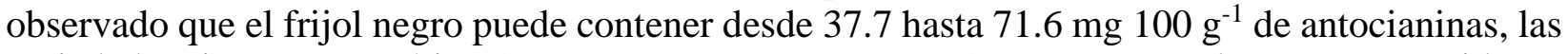
variedades tipo Mesoamérica (grano pequeño y testa opaca) presentaron el mayor contenido en grano y testa, por lo que pueden usarse como antioxidantes naturales, porque son principalmente del tipo no acilado (Salinas-Moreno et al., 2005)

La mezcla de sabores y texturas estimula el paladar de los consumidores. En los vegetales el sabor dulce es el resultado de su contenido de azúcares (Sims y Golaszewski, 2003). Al ingerir azúcares como glucosa y fructosa se activan diferentes receptores (Lee y Owyang, 2017). Los azúcares no solo afectan el sabor, sino que también pueden alterar la percepción de sabores asociados con otros compuestos orgánicos (Auerswald et al., 1999). Pruebas con panelistas han demostrado que se prefieren las variedades de $P$. vulgaris clasificadas como dulces e incluso utilizaron la dulzura para diferenciar entre cultivares (Mkanda et al., 2007; Wszelaki et al., 2005). 
VandenLangenberg et al. (2012), refieren diferencias significativas entre genotipos de frijol ejotero, tanto en la cantidad de azúcares, así como en los patrones de acumulación de azúcares; mientras que no observaron interacciones entre genotipo*año de cultivo para las variables mencionadas. En la variedad de frijol común Puebla 152 VandenLangenberg et al. (2012), encontraron que conforme avanzó el desarrollo de la planta el contenido de sacarosa se mantuvo estable, mientras que la fructosa y la glucosa se incrementaron en forma lineal.

Con estos antecedentes el objetivo del presente estudio fue conocer el contenido de azúcares totales y de proteína en compuestos de frijol nativo provenientes de la región Triqui de Oaxaca, sembradas para autoconsumo.

\section{Material biológico}

Se utilizaron tres muestras compuestas de semilla de frijol provenientes de Santo Domingo del Estado, Oaxaca, las cuales fueron colectadas en el 2016 en hogares donde se siembran mezclas de frijol con diferentes colores y tamaños y la utilizan para autoconsumo. A cada muestra se le asignó un número (14 979, 14981 y 14983$)$.

\section{Parámetros morfológicos de la semilla}

Se pesaron $200 \mathrm{~g}$ de cada muestra compuesta y se agruparon por color y tamaño de grano Cada compuesto se dividió en submuestras en función de su tamaño y color.

\section{Color}

Se determinó de forma visual y se separaron para su análisis las semillas que constituían más de $10 \%$ en cada muestra, por ser las más representativas.

\section{Peso y tamaño}

Se midió el peso de 100 granos, los cuales se seleccionaron al azar y se pesaron en una balanza semianalítica digital. Se clasificaron como granos pequeños aquellos con un peso menor de $25 \mathrm{~g}$ 100 semillas $^{-1}$, medianos aquellos cuyo peso fue de 25 a $40 \mathrm{~g} 100$ semillas $^{-1}$ y grandes cuando su peso fue superior a $40 \mathrm{~g} 100$ semillas $^{-1}$ (Allende, 2006).

\section{Análisis del contenido de azúcares totales y de proteína cruda}

Los análisis se realizaron por duplicado. El contenido de azúcares totales se determinó utilizando el método de antrona (Yemm y Willis, 1954). La lectura en el espectrofotómetro UV-VIS se realizó a $630 \mathrm{~nm}$. El contenido de proteína se determinó de acuerdo con el método de Kjeldahl empleando el equipo semiautomatizado Kjeltec-1030. El porcentaje de proteína se calculó a partir del nitrógeno total utilizando el factor 6.25 .

Los datos se analizaron bajo un diseño completamente al azar, tres tratamientos (muestras compuestas) y de dos a tres submuestras, cada una con dos repeticiones. Se usó el procedimiento Modelo Lineal General (GLM) del Sistema de Análisis Estadístico versión 8 (SAS Institute, 1999). La comparación de medias se realizó con la prueba de Tukey $(p \leq 0.05)$. Además, se obtuvo una matriz de correlaciones de Pearson. 
En el Cuadro 1 se muestra el color de las submuestras de mayor a menor predominancia de los granos de frijol en las que se subdividió cada muestra compuesta, así como también el peso de 100 semillas, el contenido de proteína y de azúcares totales.

Cuadro 1. Características físicas, contenido de proteína y azúcares en tres muestras compuestas de frijol de la Región Triqui alta de Oaxaca.

\begin{tabular}{|c|c|c|c|c|c|}
\hline $\begin{array}{l}\text { Número } \\
\text { de } \\
\text { muestra }\end{array}$ & Color/especie & $\begin{array}{c}\text { Proporción } \\
(\%)\end{array}$ & $\begin{array}{l}\text { Peso de } 100 \\
\text { semillas }(\mathrm{g})\end{array}$ & $\begin{array}{l}\text { Proteína } \\
(\%)\end{array}$ & $\begin{array}{c}\text { Azúcares } \\
\text { totales }\end{array}$ \\
\hline \multirow[t]{5}{*}{14979} & Amarillo (P. vulgaris L.) & 55.9 & 32.7 & 24.8 & 6.5 \\
\hline & Negro (P. vulgaris L.) & 19.2 & 31.3 & 23.7 & 6.4 \\
\hline & Rojo oscuro (P. vulgaris L.) & 13.7 & 30.1 & 24.4 & 6.4 \\
\hline & Pinto beige/negro (P. vulgaris L.) & 6.3 & 31.8 & nd & nd \\
\hline & Beige (P. vulgaris L.) & 4 & nd & nd & nd \\
\hline \multirow[t]{4}{*}{14981} & Rojo $(P$. coccineus $)$ & 73.8 & 73.3 & 23.4 & 9.5 \\
\hline & Amarillo (P. vulgaris L.) & 15.8 & 25.3 & 23.3 & 6.2 \\
\hline & Negro (P. vulgaris L.) & 8.5 & 34.7 & nd & nd \\
\hline & Pinto beige & 1.9 & 20 & nd & nd \\
\hline \multirow[t]{4}{*}{14983} & Negro (P. coccineus) & 76.8 & 70 & 21.7 & 9.6 \\
\hline & Pinto vino ( $P$. coccineus $)$ & 10.3 & 61.3 & 23.5 & 11.6 \\
\hline & Vino $(P$. coccineus $)$ & 7.9 & 70.1 & nd & nd \\
\hline & Beige $(P$. coccineus $)$ & 5 & 64.2 & nd & nd \\
\hline
\end{tabular}

nd $=$ dato no disponible; ${ }^{*}=$ dato en base seca.

En cada muestra compuesta se identificaron de cuatro a cinco diferentes colores y tamaños de

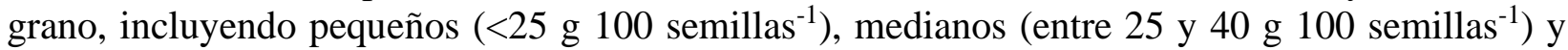
grandes (>40 g 100 semillas $\left.^{-1}\right)$. Los granos pequeños y medianos se identificaron como frijol común (Phaseolus vulgaris L.), mientras que los grandes se identificaron como ayocote (Phaseolus coccineus L.). Los frijoles negros y rojos $P$. coccineus, así como los amarillos y negros de $P$. vulgaris, fueron los más frecuentes dentro de las tres muestras compuestas; seguidos por los pintos, vino y beige; estos últimos representaron una proporción menor al $10 \%$ en las muestras.

El peso de grano varió desde $20 \mathrm{~g} 100$ semillas $^{-1}$ hasta $73.3 \mathrm{~g} 100$ semillas $^{-1}$. Se detectaron diferencias estadísticas altamente significativas entre las muestras compuestas $(p \leq 0.01)$ para la variable peso de 100 semillas. La muestra 14979 estuvo conformada por $P$. vulgaris de tamaño mediano, aunque de diferentes colores, con un peso entre 30 y 33 g 100 semillas $^{-1}$. El tamaño de grano predominante en dos de las muestras fue el grande (>40 g 100 semillas $\left.^{-1}\right)$, por tratarse de $P$. coccineus, que representó 74\% de la 14981 y el 100\% de la muestra 14 983; Los granos de tamaño pequeño fueron poco frecuentes y representaron menos de $2 \%$ de la muestra 14981.

La muestra 14979 contenía granos de color amarillo, negro, rojo y pinto. Tres de los cuatro colores que la conformaron son similares a los reportados por Espinosa-Pérez et al. (2015), para la colecta 040 de Oaxaca, la cual es tipo Ensaladilla con frijoles de color de testa negra, roja y pinto y su

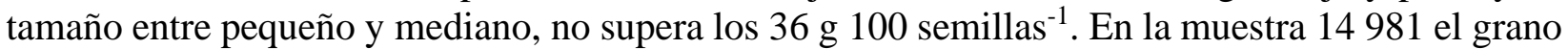
rojo $P$. coccineus (73.3 g 100 semillas $^{-1}$ ), fue el predominante, y $24 \%$ de la muestra estuvo constituido por $P$. vulgaris amarillos y negros de tamaño mediano (25.3 y 34.7 g 100 semillas $^{-1}$ 
respectivamente) y únicamente un $2 \%$ de color pinto-beige $P$. vulgaris tamaño pequeño (20 g 100 semillas $^{-1}$ ). Por último, en la muestra 14983 los cuatro granos de frijol fueron $P$. coccineus, un $77 \%$ color negro y $23 \%$ de colores pinto, vino y beige.

\section{Contenido de azúcares totales}

El porcentaje de azúcares totales en los tipos de grano fue de 6.2 a $6.5 \%$, en los granos $P$. vulgaris, clasificados de tamaño mediano, mientras que en los $P$. coccineus, clasificados de tamaño grande, los azúcares representaron del 9.5 al $11.7 \%$. Se detectaron diferencias estadísticas altamente significativas entre las muestras compuestas $(p \leq 0.01)$ en el contenido de azúcares totales. La concentración de azúcares totales se correlacionó positivamente con el tamaño de grano $\left(\mathrm{r}=0.88^{* *}\right)$.

Se observó variación en el contenido de azúcares de los diferentes colores de grano; sin embargo, las diferencias más notables se observaron entre las dos especies: $P$. vulgaris y $P$. coccineus. También en frijol ejotero, se han observado diferencias entre genotipos en la cantidad de azúcares totales, mientras que en el frijol común Puebla 152 se observó que conforme avanzó el desarrollo de la planta se modificó la concentración de algunos azúcares; el contenido de sacarosa se mantuvo estable, mientras que la fructosa y la glucosa se incrementaron (VandenLangenberg et al., 2012).

El mayor contenido de azúcares en $P$. coccineus en comparación con $P$. vulgaris, se asocia con sabor más dulce, lo cual de acuerdo a los trabajos de (Mkanda et al., 2007; Wszelaki et al., 2005), puede motivar una mayor aceptación por parte de los consumidores. Lo anterior, se reafirma con testimonios de las mujeres de Santo Domingo del estado, quienes afirmaron que el frijol ayocote negro y rojo son los de mejor sabor, por ello son parte de los platillos tradicionales; se usan para hacer atole de frijol (nakinj runee); uno de los platillos típicos de la región triqui alta, se puede consumir en día de muertos y en los velorios (Martínez-López et al., 2018). En la Figura 1 se muestra el contenido de azúcares totales y el tamaño de grano de cada submuestra de frijol en las tres muestras compuestas.

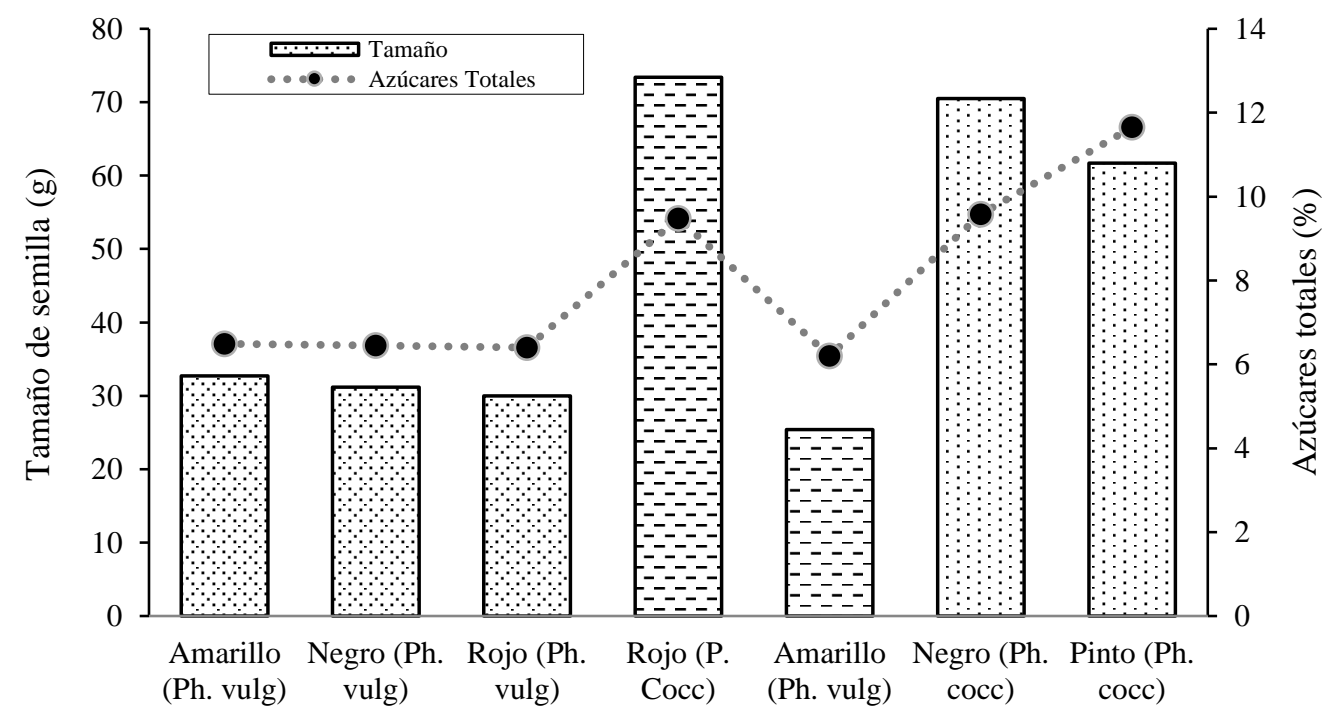

Figura 1. Contenido de azúcares totales y tamaño del grano de tres compuestos de frijol $(P$. vulgaris y $P$. coccineus). Cada textura de barra agrupa las submuestras que conforman un compuesto. 
Las diferencias en el contenido de azúcares totales entre los $P$. vulgaris y los $P$. coccineus que componen la muestra, sugieren que cada una de las muestras, de acuerdo a la predominancia de una u otra de estas especies de frijol tendrá mayor o menor intensidad de sabor dulce y con ello sus atributos organolépticos serán distintos. El contenido de proteína mostró diferencias estadísticas significativas entre las muestras compuestas $(p \leq 0.05)$. La variación entre submuestras fue desde $21.7 \%$ en $P$. coccineus negro, hasta $24.8 \%$ en $P$. vulgaris amarillos (Cuadro 1 ).

En los genotipos de P. vulgaris la proteína se ubicó dentro del intervalo reportado para frijoles nativos de diferentes zonas de México que estuvo entre 17.8 y 26.5\% (Jacinto-Hernández et al., 2002). Se observó la tendencia a mayor contenido de proteína en los frijoles $P$. vulgaris, cuyo peso varió entre 25.3 y 34.7 g 100 semillas $^{-1}$, en comparación con los $P$. coccíneus que mostraron peso entre 61.3 a 73.3 g 100 semillas $^{-1}$.

Lo anterior contribuyó a una correlación negativa entre el tamaño de grano y el contenido de proteína $\left(\mathrm{r}=-0.61^{*}\right)$. También se observaron diferencias en el contenido de proteína entre genotipos de la misma especie. En la Figura 2 se muestra el contenido de proteína y el tamaño de grano de cada submuestra de frijol en las tres muestras compuestas.

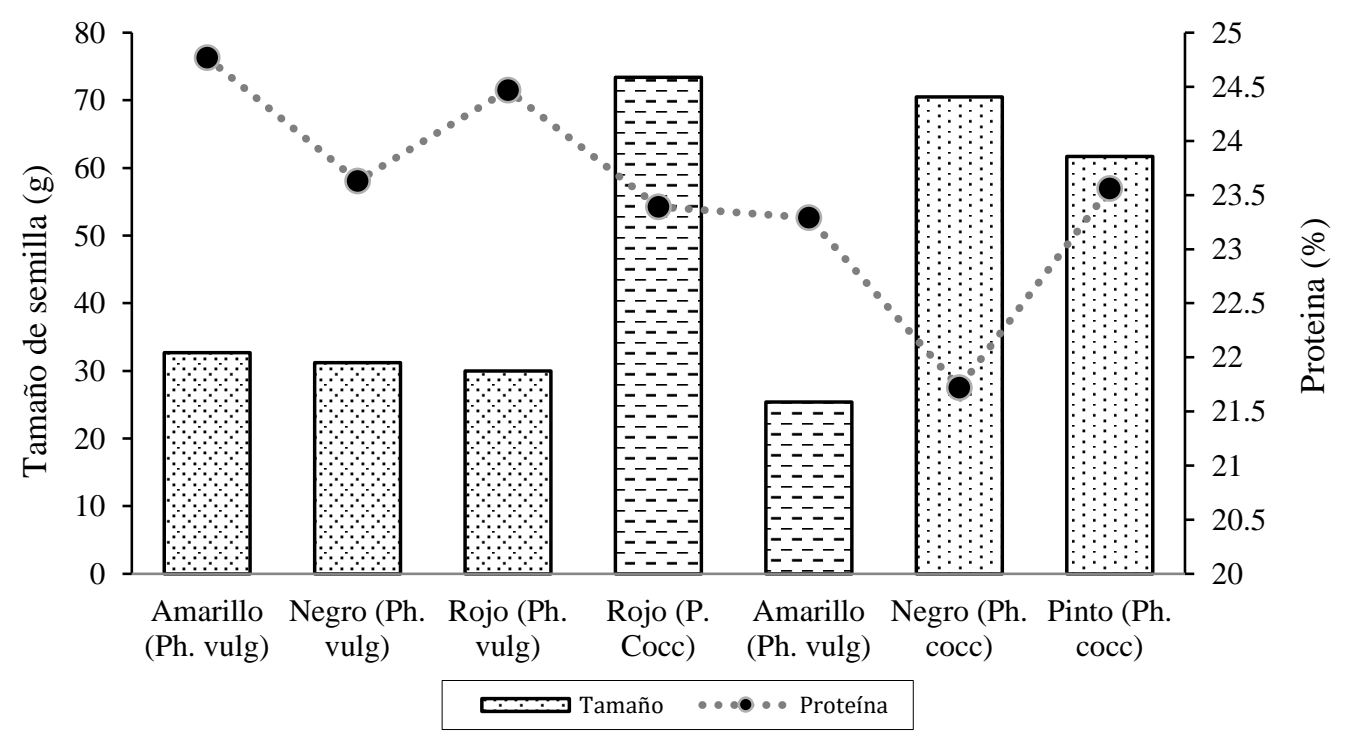

Figura 2. Contenido de proteína cruda y tamaño del grano de tres compuestos de frijol ( $P$. vulgaris y $\boldsymbol{P}$. coccineus). Cada textura de barra agrupa las submuestras que conforman un compuesto.

En la muestra compuesta 14979 el intervalo de proteína en las submuestras $P$. vulgaris varió de 23.7 a $24.8 \%$; los frijoles amarillos y rojos de tamaño mediano fueron los que tuvieron mayor contenido con 24.8 y $24.5 \%$ (Cuadro 1), mientras que en la muestra 14981 tanto $P$. coccineus como $P$. vulgaris mostraron valores cercanos, 23.3 y $23.4 \%$ respectivamente.

El compuesto 14983 mostró diferencia entre las dos muestras $P$. coccineus que lo componen negro $21.7 \%$ y pinto-vino $23.5 \%$ de proteína. El color del grano no se asoció con el contenido de proteínas y azúcares totales; Sin embargo, el tamaño de grano sí. Un tamaño de grano más grande, un mayor porcentaje de azúcares $\left(\mathrm{r}=0.88^{* *}\right)$ y un contenido de proteína más bajo $\left(\mathrm{r}=-0.61^{*}\right)$. 


\section{Conclusiones}

Se observó mayor contenido de azúcares en frijoles $P$. coccineus en comparación con $P$. vulgaris; mientras que estos últimos mostraron mayor contenido de proteína. Un mayor tamaño de grano se asoció con mayor contenido de azúcares totales $\left(\mathrm{r}=0.88^{* * *}\right)$ y menor contenido de proteína $(\mathrm{r}=$ $\left.-0.61^{*}\right)$.

La muestra compuesta 14979 , conformada por diferentes colores de $P$. vulgaris, mostró superior contenido promedio de proteína (24.3\%) mientras que la 14983 conformada por $P$. coccineus mostró el sabor más dulce (10.7\% azúcares totales), aunque inferior contenido promedio de proteína $(22.7 \%)$.

Los valores obtenidos indicaron que el color de grano no se asoció con los contenidos de proteína ni de azúcares totales.

La variación natural que tiende a existir entre el contenido de proteína cruda y azúcares totales entre $P$. vulgaris y $P$. coccineus, puede utilizarse para diseñar compuestos con diferentes característicar sensoriales y nutricionales que puedan incorporarse a mercados tanto locales en poblaciones rurales como en las urbes.

\section{Literatura citada}

Auerswald, H., D. Schwarz, C. Kornelson, A. Krumbein, and B. Bruckner. 1999. Sensory analysis, sugar and acid content of tomato at different EC values of the nutrient solution. Sci. Hort. 82(3):227-242.

Bourges, R. H. 2001. Alimentación y nutrición en México. Comercio Exterior. 51(10):897-904.

Espinosa-Pérez, E. N., Ramírez-Vallejo, P., Crosby-Galván, M. M., Estrada-Gómez, J. A., LucasFlorentino, B., \& Chávez-Servia, J. L. 2015. Clasificación de poblaciones nativas de frijol común del centro-sur de México por morfología de semilla. Rev. Fitotecnia Mexicana. 38(1):29-38.

Garzón, G. A. 2008. Las antocianinas como colorantes naturales y compuestos bioactivos: revisión. Red de Revistas Científicas de América Latina, Redalyc. Org. 13(3):27-36.

Gispert C., M., A.R. Gonzáles E., H. Rodríguez G., L. Luna C., I. de la Cruz C. I. 2004. La montaña de Humo. Tesoros Zoques de Chiapas. $1^{\text {a }}$ ed. Universidad Nacional Autónoma de México (UNAM). $101 \mathrm{p}$.

Jacinto-Hernández, C., Bernal-Lugo, I. Garza-García, R., 2002. Food quality of drybean (Phaseolus vulgaris L.) landraces from different states of México. Annual Report of the Bean Improvement Cooperative BIC. Michigan State University. East Lansing, MI 48824. 45(1):222-223.

Lee, A. A., and Owyang, C. 2017. Sugars, Sweet Taste Receptors, and Brain Responses. Nutrients, MDPI, 9(7):653-685.

Martínez-López, L., Zapata-Martelo, E., Ayala-Carrillo, M. d., Martínez-Corona, B., VázquezCarrillo, G., Jacinto-Hernández, C., y Espinosa-Calderón, A. 2018. Conocimiento práctico y teórico de maíz y frijol en la Región Triqui Alta, Oaxaca. Revista Mexicana de Ciencias Agrícolas. 9(1):111-121.

Martínez-López, L. 2018. Comunicación personal. 21 de noviembre de 2018. 
Mkanda, A.V., A. Minnaar, and H.L. de Kock. 2007. Relating consumer preference to sensory and physicochemical properties of dry beans (Phaseolus vulgaris). J. Sci. Food Agr. 87(15):2868-2879.

Muñoz-Velázquez, E. E., Rubio-Hernández, D., Bernal-Lugo, I., Garza-García, R., y JacintoHernández, C. 2009. Caracterización de genotipos nativos de frijol del estado de Hidalgo, con base a calidad del grano. Agricultura Técnica en México. 35(4):429-438.

Ramos R., R. M., \& Sandoval M., K. 2007. Estado nutricional en la marginación y la pobreza de adultos triquis del estado de Oaxaca, México. Rev. Panam. Salud Pública. 22(4):260-267.

Salinas-Moreno, Y., Rojas-Herrera, L., Sosa-Montes, E., y Pérez-Herrera, P. 2005. Composición de antocianinas en variedades de frijol negro (Phaseolus vulgaris L.) cultivadas en México. Agrociencia. 39:385-394.

Sims, C. A. and R. Golaszewski. 2003. Vegetable flavor and changes during postharvest storage. In: Bartz, J.A. and J.K. Brecht (eds.). Postharvest physiology and pathology of vegetables. Marcel Dekker, New York, NY. 331-340 pp.

Statistical Analysis Systems Institute (SAS Institute). 1999. The SAS for windows version eight. SAS Institute Inc. Cary, N. C., USA. 1028 p.

VandenLangenberg H. M. Bethke, P. C. Nienhuis, J. 2012. Patterns of fructose, glucose, and sucrose accumulation in snap and dry bean (Phaseolus vulgaris) pods. HortScience. 47(7):874-878.

Wszelaki, A.L., Delwiche, J.F., Walker, S.D., Liggett, R.E., Miller, S.A. and Kleinhenz. M.D. 2005. Consumer liking and descriptive analysis of six varieties of organically grown edamametype soybean. Food Qual. Prefer. 16(8):651-658.

Yemm, E. W. and A. J. Willis. 1954. The estimation of carbolhy-drates in plant extracts by anthrone. Biochem. J. 57(1):508-514. 